\title{
Experimental study of wind impact on multi- level industrial scaffolding
}

\author{
Lyudmila Frishter ${ }^{1, *}$, and Maxim Lukin ${ }^{1}$ \\ ${ }^{1}$ Moscow State University of Civil Engineering, 129337, Yaroslavskoye Shosse 26, Moscow, Russian \\ Federation
}

\begin{abstract}
In the article, the local stress-strain state of structures and constructions is investigated, various variants for the design of the boundary are taken into account: special lines, points. The acting forced deformations don't satisfy the compatibility conditions. They have a finite discontinuity along the contact line (surface) of the domains, including the irregular point of the boundary, causing stresses. The subject of article is stress concentrators - the singularity of the stress-strain state of structures and constructions exhibiting "constructive heterogeneity" and discontinuous forced deformations determined on polymer models of photoelasticity and defrosting of deformations. A complex theoreticalnumerical-experimental approach, for obtaining and analyzing the stress state in the neighborhood of the irregular point of the plane domain boundary, is proposed to extrapolate reliable experimental data to a domain where the fringe contour is not readable.
\end{abstract}

\section{Introduction}

The aerodynamic aspects of the framework type structures are important in the design of buildings, calculation of air flows inside the building, evaluation of a building's influence on the air flow mode of the neighborhood, optimum selection of enclosures [1-9]. The calculation of the wind impact influence during the design of framework-type structures is guided by codes having much in common [10]. The codes do not show the protection shielding procedure, the analysis of the wind impact on the structure for different arrangement conditions - angle of attack of the wind impact on the structure. During the design of such structures, the codes prescribe dummy testing in architectural and civil wind tunnels [10-16].

Objectives of the paper: carrying out of experimental studies of the wind impact on open and shielded framework-type structures of rectangular layout for various impact angles of the wind flow; analysis of the influence of the wind flow angle of attack on the internal forces and aerodynamic factors of open and closed framework-type structures.

It is the purpose of the present paper to analyze the wind impact on the force and torque specifications of a framework-type structure for different wind angles of attack on the structure. The relevance of the paper is stipulated by the necessity of determining optimum

*Corresponding author: 1frishter@mail.ru 
functioning conditions under the wind impact both for safe and comfortable working of people, and for the operational reliability of the process equipment within the structure.

The analyzed framework-type structure under the wind impact is rectangular by layout. External framework-type structures are used for construction of high-rise process facilities, for process equipment erection, as storage buildings. Due to the complex wind flow pattern inside the open scaffolding and the necessity for a comfortable working / operation environment for people / equipment, variants of structures fully open to and fully screened against the wind are considered.

The structure has the length $l=\xi$, the height $H=3 / 4 \xi$, the width $b=5 / 8 \xi$, where $\xi$ is the characteristic size. The drawing of the structure is shown in Fig. 1. The structure is regularly pitched longitudinally: $l_{0}=l / 4=\xi / 4$, in the height $h_{0}=h / 5=\xi / 8$, transversally $b_{0}=b / 5=\xi / 8$.
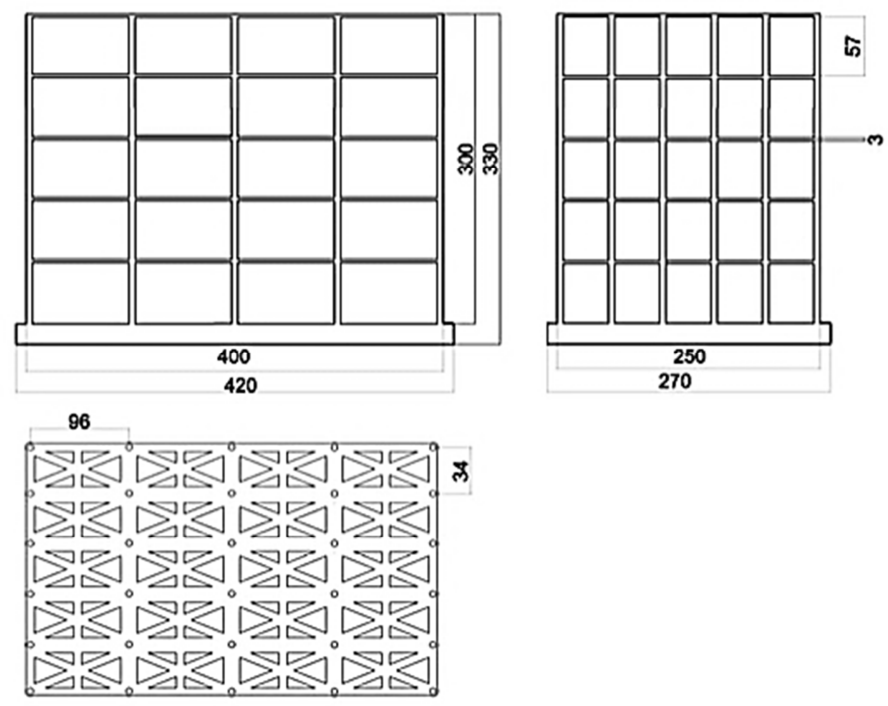

Fig. 1. Drawing of a framework-type structure: frontal view, top view, side view.

The angle between the impact vector of the approach wind flow and the frontal surface of the structure, called "angle of attack", is variable. This paper handles a method of experimental modeling of the wind impact on the industrial scaffolding [8-14]. Different positions of the structure are studied experimentally at a constant wind direction. This allows for an analysis of the influence of the angle of attack of the wind flow on the internal forces and aerodynamic factors of the structure. For a framework-type structure, the experimental data will allow for recommendations on scaffolding installation under recognition of the wind rose in an optimum manner for its functioning $[2,4,9,10,14-16]$.

\section{Experimental aerodynamic studies of a framework-type structure}

\subsection{Problem statement. Theoretical provisions of experimental problem solution}

The wind impact on the framework-type structure is studied experimentally, in a wind tunnel. A model of the structure is mounted in the section of the wind tunnel with flat and 
steady air flow. Due to the stationarity of the flow, all components containing particular time derivatives in the equation of motion and continuity are equal to zero. The flat and steady flow can be considered as a test mode of gas dynamics. No gradient surface pressure measurement on the structure was conducted along the height.

Flat steady flows without recognition of volume forces suit the following resolving system of equations [3]: continuity equation:

$$
\frac{\partial\left(\rho V_{x}\right)}{\partial x}+\frac{\partial\left(\rho V_{y}\right)}{\partial y}=0
$$

momentum conservation equation:

$$
\frac{1}{\rho} \frac{\partial p}{\partial x}+\frac{\partial}{\partial x}\left(\frac{V^{2}}{2}\right)=V_{y}\left(\frac{\partial V_{y}}{\partial x}-\frac{\partial V_{x}}{\partial y}\right), \frac{1}{\rho} \frac{\partial p}{\partial y}+\frac{\partial}{\partial y}\left(\frac{V^{2}}{2}\right)=-V_{x}\left(\frac{\partial V_{y}}{\partial x}-\frac{\partial V_{x}}{\partial y}\right),
$$

energy conservation equation:

$$
\frac{d}{d t}\left(\frac{p}{\rho^{\gamma}}\right) \equiv V_{x} \frac{\partial}{\partial x}\left(\frac{p}{\rho^{\gamma}}\right)+V_{y} \frac{\partial}{\partial y}\left(\frac{p}{\rho^{\gamma}}\right)=0,
$$

where $V$ is the flow velocity, $V=\sqrt{V_{x}^{2}+V_{y}^{2}}, V_{x}, V_{y}$ are flow velocity components along $O X, O Y$ respectively, $p, \rho$ is the gas (air) flow pressure, density, $\gamma$ is the isentropic exponent $\left(\gamma=\frac{c_{p}}{c_{v}}\right), c_{p}, c_{v}$ are specific thermal capacities of the gas mixture.

For a vortex-free flow:

$$
\frac{\partial V_{y}}{\partial x}-\frac{\partial V_{x}}{\partial y}=0
$$

Then, in case of a vortex-free stationary mono-directional flow and in absence of mass forces, the obtained momentum equations are reduced and converted to the Bernoulli equation:

$$
\rho v d v=d p \text { or } p=\rho v^{2} / 2+p_{0} .
$$

Experimental studies of the air flow impact on the structure are conducted in the flow area where a flat steady stream can be reduced to mono-directional vortex-free steady flow. The wind flow producing an impact on the structure, and the angle of attack of the wind flow are considered constant over the height, the length and the width of the external surface of the structure.

During a scale model test in the wind tunnel, no match can be achieved with the fullscale Reynolds number [11-14]. For structures of such class (poorly streamlined bodies), there is an intrinsic "auto-modeling range by Reynolds number $R e$ ", when with the increase of $R e$ number, the dimensionless body flow-around specifications almost cease to depend on this parameter [3, 11-14]. To determine the auto-modeling range by Reynolds number for the model under study, auxiliary tests were carried out at sequential increasing of the flow velocity in the tunnel from $V_{\infty}=$ from 5 to $30 \mathrm{~m} / \mathrm{s}$. The obtained dependencies allow for a conclusion that for the present model auto-modeling by Reynolds number at $V_{\infty} \geq 7 \mathrm{~m} / \mathrm{s}$ is present $[3,9,11-14]$. 
The experiment handles the case when a unidirectional wind flow encounters an obstacle - the framework-type structure. The aerodynamic factors for the longitudinal, the normal and the transversal forces are determined like [10]:

$$
C_{x}=\frac{F_{x}}{q_{\infty} S}, \quad C_{y}=\frac{F_{y}}{q_{\infty} S}, \quad C_{z}=\frac{F_{z}}{q_{\infty} S}
$$

respectively.

The interaction of the flow with the structure is complicated by the open internal spaces of the framework-type structure.

\subsection{Design and making of the model}

For experimental studies, a model of industrial scaffolding was developed and made. A dummy structure with rectangular columns network measures in the layout $250 \mathrm{x} 400 \mathrm{~mm}$, the height is equal to $300 \mathrm{~mm}$. The permeable floor decks are evenly distributed all-over the height of the structure with $60 \mathrm{~mm}$ vertical pitch, beginning with the zero level up to the upper elevation mark of the structure. Under recognition of the test section size of the wind tunnel, the largest scale of the model of 1:100 is selected. The dummy is made of M6 threaded steel rods imitating the vertical columns. The floor decks and their parts (levels 15) are made of acrylic glass $3 \mathrm{~mm}$ thick by means of Qualitech 9G 530 IV CMC laser cutting machine, of a solid workpiece. The steel rods were screwed into a sheet of veneer being the baseplate of the dummy. After that, the acrylic glass sheets with pre-drilled holes were pulled over the threaded rods.

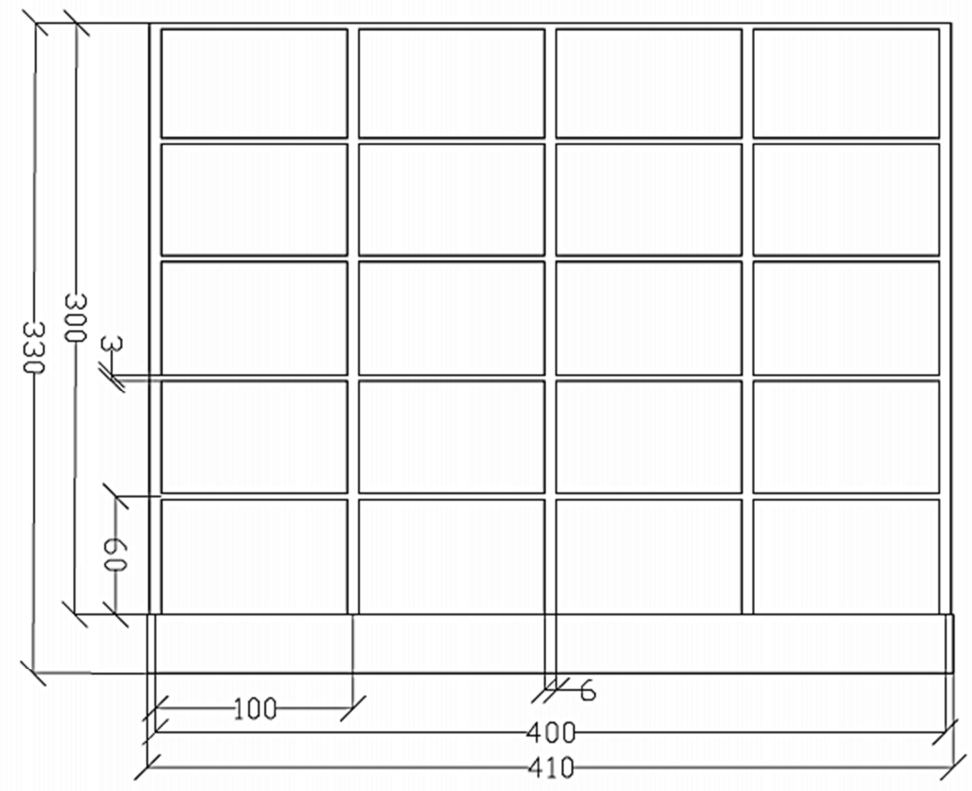

Fig. 2. Scaffolding dummy diagram, side view. 


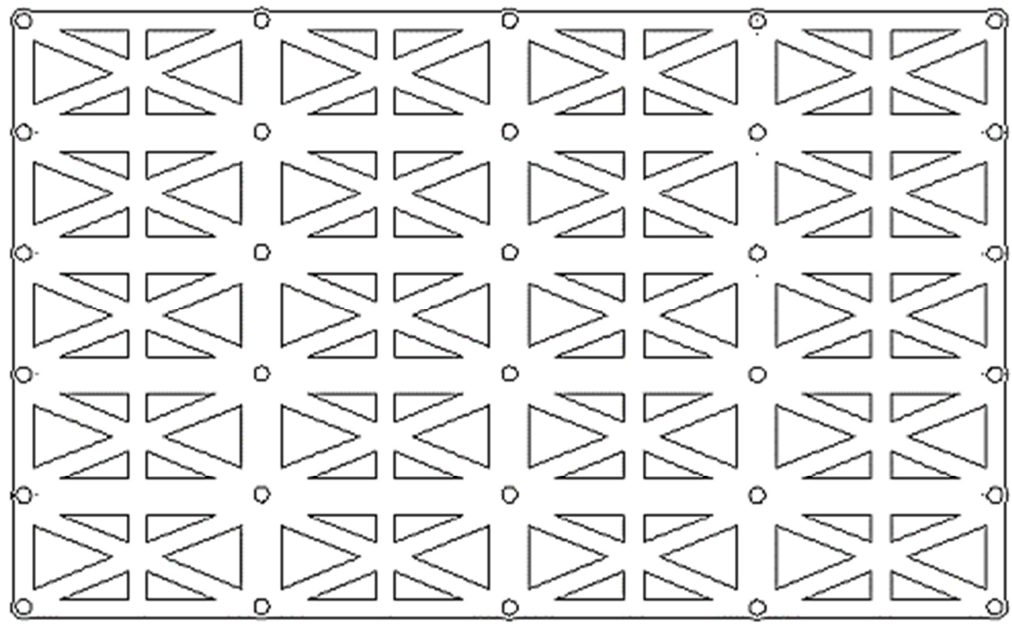

Fig. 3. Scaffolding floor decks diagram (levels 1-5)

After the final installation of all required parts, a final measurement of all dimensions of the industrial scaffolding dummy was made (Fig. 4).

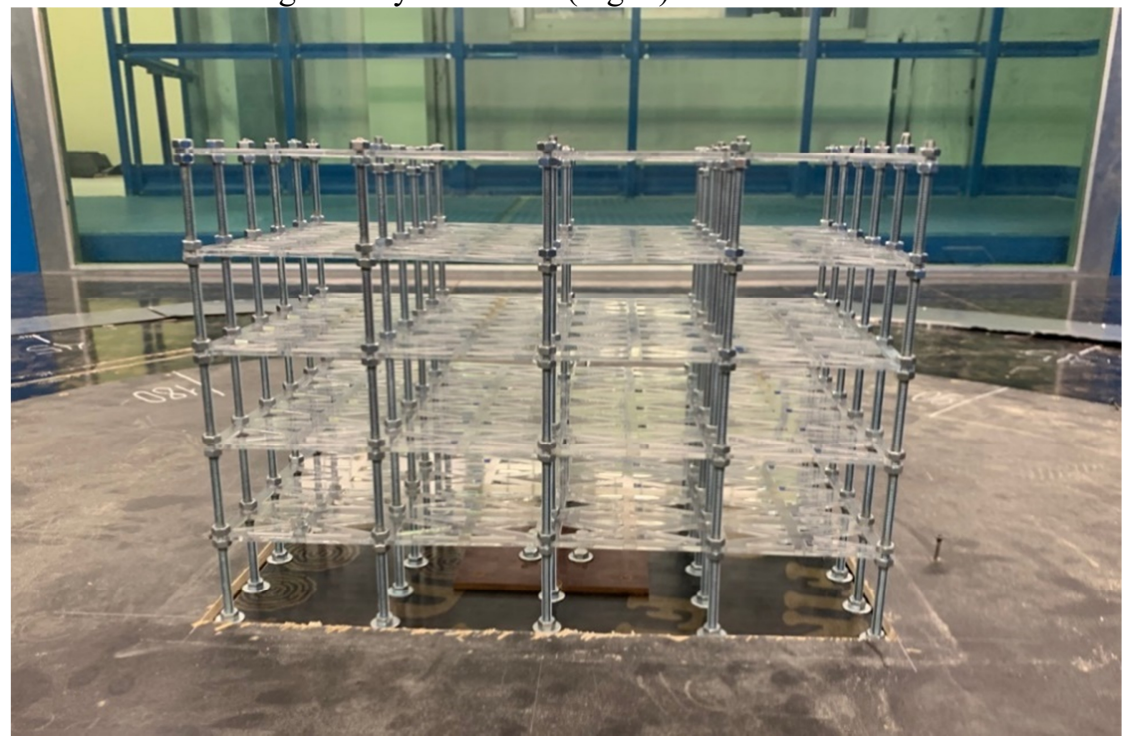

Fig. 4. Industrial scaffolding dummy. Side view.

During the model building stage, a 6-component force and torque sensor block was built-in for measurement of the resulting aerodynamic forces and torques. The sensor is fastened to a rigid metal plate in the base of the dummy. The assembled dummy was put on an automatic rotary table in the testing section of the wind tunnel.

\subsection{Test fixture and measurement instruments}

The experiment has been conducted in a specialized wind tunnel of the Academic, Scientific and Manufacturing Laboratory of Aerodynamics and Acoustics (UNPL AAISK NIU MGSU). This plant has a closed-circuit flow path and a modular fan unit of nine 
modules. The axial length of the closed-circuit flow path is $96 \mathrm{~m}$, the testing section of the tunnel is $18.9 \mathrm{~m}$. The length of the testing section allows for modeling the ground layer of the atmosphere in different conditions. The measurement is made at a flow velocity of $\mathrm{V} \infty$ $=12 \mathrm{~m} / \mathrm{s}$. The dimensionless aerodynamic factors of the scale model obtained during the aerodynamic experiment are similar to the respective values in full-scale conditions [1-6, 16]. For force and torque measurements, Schunk FTD load cells were used, making-up a compact 6-component sensor block. The collected data are processed by means of ATI Software, as a result, the values of the forces and moments are computed. The calibration table was provided by the load cell manufacturer.

\subsection{Experimental aerodynamic studies of the wind load impact on the model}

A dummy industrial scaffolding is put into the testing section of the wind tunnel on a rotary table, which allows for studying the influence of the wind impact direction change (angles of attack of the flow) on the structure. In the beginning of the experiment, the permeable structure is put into the position of the angle of attack $\beta=0^{0}$, and the "reference" (no air flow) readings of the load cell is recorded, to account for the initial displacement when the load cell takes just the weight of the model structure. By means of the model bench rotation in the wind tunnel, the angle of attack is changed from $0^{\circ}$ to $360^{\circ}$ with a pitch of $45^{\circ}$ (Fig. 5). In the course of the testing, for each angle of attack of the flow to the model, the readings of the load cells are recorded. Then, wind-protection shields are mounted on the model, and the readings of the load cells are recorded, again, for each of the angles of attack of the wind impact.

By means of the six-component load cell blocks and ATI DAQ F/T Software, the following readings are obtained $F_{x}, F_{y}, F_{z}$ and - total forces (tf), torque $M_{z}$ along $Z$-axis and bending torques $M_{x}, M_{y}$ along $X$-, $Y$-axis, respectively, and by formulas (1), the following values are calculated $C_{x}, C_{y}, C_{z}, C M_{x}, C M_{y}, C M_{z},-$ total aerodynamic resistance factors relative to $X, Y$ and $Z$ axes, respectively.

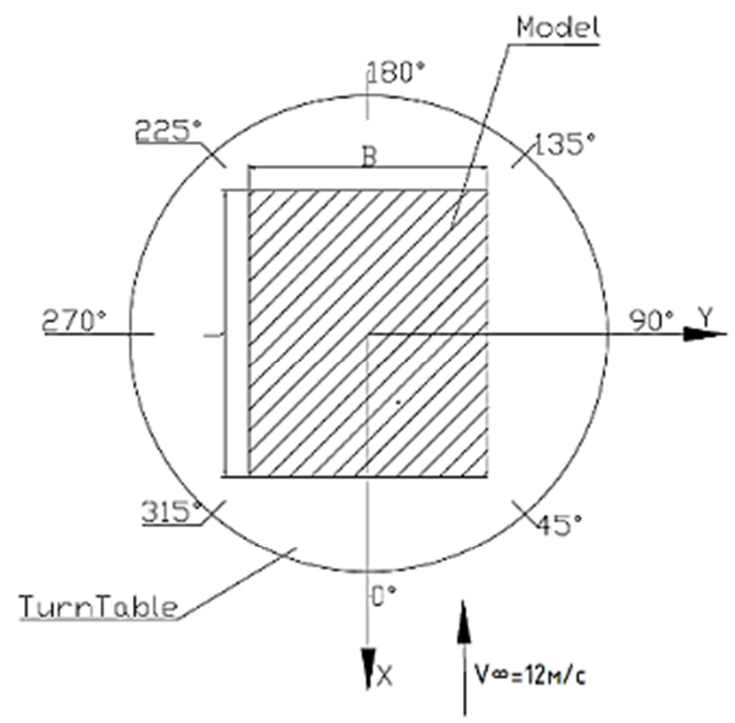

Fig.5. Fixture schematic of the rotary table with swing angles and the resting test object. 


\section{Results of the experiment. Analysis of obtained data}

Table 1 shows forces $F_{x}, F_{y}, F_{z}$ and torques $M_{x}, M_{y}, M_{z}$ based on the load cell readings in the baseplate of the model at changing of the angle of attack $\beta$ of the wind flow from $0^{\circ}$ to $360^{\circ}$ with a pitch of $45^{\circ}$ for fully open framework-type structure $(0000)$.

Table 1. Experimental data of aerodynamic impacts for the open structure (variant 0000)

\begin{tabular}{|c|c|c|c|c|c|c|}
\hline \multirow{2}{*}{$\begin{array}{c}\text { Angle } \\
\text { of } \\
\text { attack } \\
\beta\end{array}$} & \multicolumn{6}{|c|}{ Force and torque readings } \\
\cline { 2 - 7 } & $F_{x}$ & $F_{y}$ & $F_{z}$ & $M_{x}$ & $M_{y}$ & $M_{z}$ \\
\hline 0 & 1.749 & 4.78 & 2.026 & -0.29 & 0.003 & -0.0066 \\
\hline 45 & -1.87 & 4.25 & 2.65 & -0.249 & -0.279 & 0.009 \\
\hline 90 & -4.21 & -0.73 & 0.44 & 0.072 & -0.34 & -0.01 \\
\hline 135 & -4.75 & -5.039 & 1.61 & 0.366 & -0.273 & -0.008 \\
\hline 180 & -2.92 & -5.58 & 0.92 & 0.39 & 0.06 & -0.011 \\
\hline 225 & 2.78 & -4.34 & 0.272 & 0.3 & 0.318 & 0.011 \\
\hline 270 & 4.41 & 0.31 & 0.118 & 0.0008 & 0.34 & -0.008 \\
\hline 315 & 5.09 & 4.71 & 1.81 & -0.28 & 0.26 & -0.008 \\
\hline
\end{tabular}

The obtained values of the torque specifications demonstrate that the torque $M_{z}$ equals to zero, that is stipulated by the symmetry of the structure, the impacts, as well as by the location of the load cells in the center of the baseplate in the first level of the structure along $O Z$ symmetry axis of the structure. The bending torques $M_{x}, M_{y}$ are also almost equal to zero.

The values $F_{x}, F_{y}$ at the angles of $135^{\circ}$ and $315^{\circ}$ are almost equal to each other, and their dependence diagrams vs. the angle of attack of the flow are similar (Fig. 6).

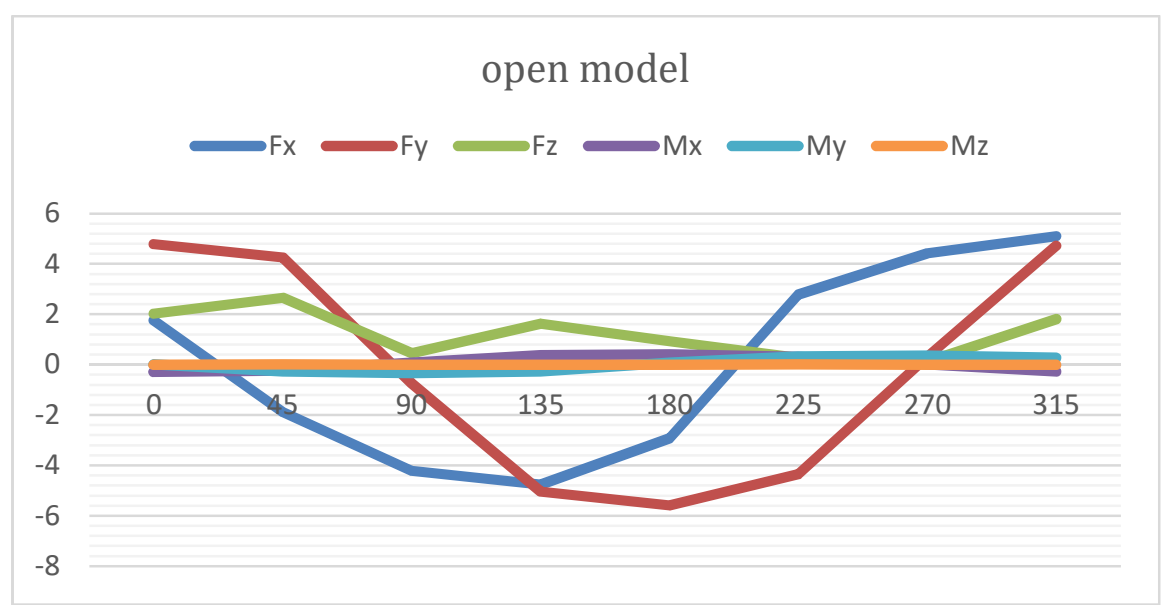

Fig.6. Diagram of the aerodynamic impact at angle of attack changed from $0^{\circ}$ to $360^{\circ}$ for the open model

The values $F_{z}$ are considerably less than the values $F_{x}, F_{y}$. The value $F_{z}=2.65 \mathrm{~N}$ exceeds other $F_{z}$ values, have no similar value at a symmetrical swing of the structure, that is obviously due to the measurement error. The biggest value of module $F_{y}=5.1 \mathrm{~N}$ is observed at the angles $315^{0}\left(135^{\circ}\right)$. The biggest value of module $F_{y}=5.6 \mathrm{~N}$ is observed for 
the angles $180^{\circ}\left(0^{\circ}\right)$, to these values, the values $F_{y}$ at the angles $135^{\circ}\left(315^{\circ}\right)$ are close. The biggest values of forces $F_{y}$ are observed for the structure at the angles $135^{\circ}$ and $315^{\circ}$ to the flow along the long windward side.

Table 2 shows force $F_{x}, F_{y}, F_{z}$ and moment $M_{x}, M_{y}, M_{z}$ values by the data of the load cell block in the baseplate of the model structure while changing the angle of attack $\beta$ of the wind flow from $0^{\circ}$ to $360^{\circ}$ with $45^{\circ}$ pitch, shielded all-around (1111).

Table 2. Experimental aerodynamic impact data for closed structure (variant 1111)

\begin{tabular}{|c|c|c|c|c|c|c|}
\hline $\begin{array}{c}\text { Angle } \\
\text { of } \\
\text { attack } \\
\beta\end{array}$ & $F_{x}$ & $F_{y}$ & $F_{z}$ & $M_{x}$ & $M_{y}$ & $M_{z}$ \\
\cline { 2 - 7 } & 3.61 & 12.84 & 7.78 & -0.738 & -0.025 & 0.036 \\
\hline 0 & $-2,54$ & 12,1 & 7.52 & -0.652 & -0.39 & 0.091 \\
\hline 45 & -6.43 & -0.19 & 5.59 & 0.014 & -0.46 & 0.059 \\
\hline 90 & -7.03 & $-12,74$ & 3.9 & 0.66 & -0.301 & -0.12 \\
\hline 135 & -3.5 & -13.17 & 3.47 & 0.75 & -0.054 & 0.053 \\
\hline 180 & 3.58 & -11.03 & 3.46 & 0.609 & 0.41 & 0.058 \\
\hline 225 & 6.37 & 2,63 & 5.55 & -0.13 & 0.44 & 0.059 \\
\hline 270 & 8.98 & 12,41 & 7.32 & 0.71 & 0.32 & -0.067 \\
\hline 315 & & & & & & \\
\hline
\end{tabular}

After mounting of protection shields from all four sides, the structure becomes more rigid, and the space factor influences the work of the structure. If the structure is completely closed with wind shields (1111), considerable forces $F_{z}=7.3-7.5 \mathrm{~N}$ are observed at the angles of attack $\beta=45^{\circ}, 315^{\circ}$.

Considerable forces $F_{x}=9.0 \mathrm{~N}$ are observed at the angles of attack $\beta=315^{\circ}$, as well $F_{x}=$ $-7.0 \mathrm{~N}$ at the angles of attack $\beta=135^{\circ}$. The strongest forces $F_{y}=-13.2 \mathrm{~N}$ are observed at the angles of attack $180^{\circ}$ and $F_{y}=12.8 \mathrm{~N}$ at $\beta=0^{0}$, close to these values are $F_{y}=12.1-12.7 \mathrm{~N}$ at the angles of attack $\beta=45^{\circ}, 315^{\circ}$. The torque $M_{z}$ is almost equal to zero, the bending torques $M_{x}, M_{y}$ are also negligible. Fig. 7 shows the dependencies of the force and torque data at different angles of attack of the wind impact for a fully shielded model.

\section{shielded model}

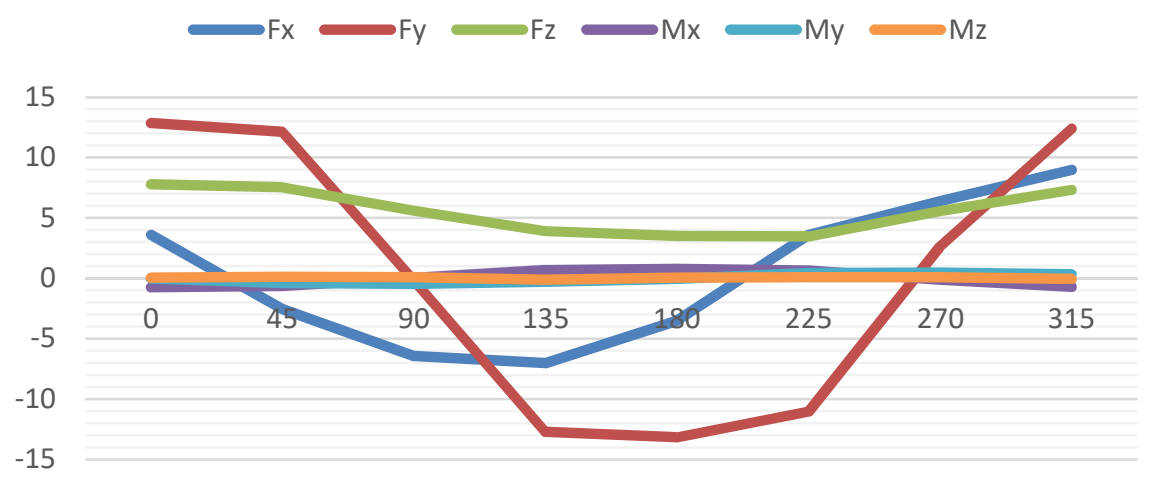

Fig.7. Aerodynamic impact vs. change of the angle of attack from $0^{\circ}$ to $360^{\circ}$ for fully shielded model

Based on the force and torque readings and by formulas (1), dimensionless aerodynamic factors were determined within the angle of attack range from $0^{\circ}$ to $360^{\circ}$ for fully open and 
fully shielded structure. The biggest aerodynamic factors $C F_{x}$ are observed for the variant of the open structure $C F_{x}\left(90^{0}\right)=-0.64$ and $C F_{x}\left(270^{0}\right)=0.67$ (in brackets: angle $\beta$ of attack of the flow). For the closed structure, these values are equal: $C F_{x}\left(270^{0}\right)=C F_{x}\left(90^{0}\right)= \pm 1.0$. The biggest values $C F_{y}$ are obtained for the open structure $C F_{y}\left(180^{0}\right)=C F_{y}\left(0^{0}\right)= \pm 0.5$. For the closed structure, the dynamics factor are, in average, $C F_{y}\left(180^{0}\right)=C F_{y}\left(0^{0}\right)= \pm(1.25 ; 1.26)$. The dynamics factor $C F_{x}$ is small for the open structure variant $(0000)$ and can be neglected. After the installation of the four shields (variant 1111), the biggest dynamics factor is $C F_{z}\left(270^{\circ}\right)=C F_{z}\left(90^{\circ}\right)= \pm 0.8$.

\section{Discussion}

The paper reports on experimental studies of the wind impact on framework-type structure. The dummy structure is fixed in the wind tunnel part with flat and steady air flow. The experimental studies of the air flow impact on the structure are conducted in the flow section where the flat and steady stream is reduced to a mono-dimensional vortex-free stationary flow, thereby, for the specific air flow velocity $V_{\infty}=12 \mathrm{~m} / \mathrm{s}$, auto-modeling by Reynolds number still applies. The air flow producing an impact on the structure and the angle of attack of the structure by the wind flow are considered constant by the height, the length and the width of the external surface of the structure. Such steady stream of the air flow against the structure can be considered as a gas-dynamics test model, which allows for the analysis of the air elasticity problem under recognition of the influence of the flow velocity direction relative to the structure. The experiment studies the influence of the position of an open and a fully shielded structure relative to the wind impact direction. Further, under recognition of the data hereof on the force and the torque specifications of the structure, as well as based on the dynamics factors, it is planned to analyze different sequences of the protection shield mounting. That allows for a selection of the structure positioning and the manner of protection shield installation optimum for functioning of the structure and comfortable working of people under a wind impact.

\section{Conclusions}

The experimental aerodynamic studies of the wind impact on a framework-type structure of rectangular layout allow for the analysis of the wind impact influence on the force and the torque specifications of the structure for different directions of the wind flow velocity and for determining of dynamic factors at different angles of attack of the wind impact on the structure. The practical significance of the paper is determined by possible recommendations on the arrangement of the framework-type structure under the wind impact for optimum functioning of the structure and comfortable working of people.

\section{References}

1. E.I. Retter, Architectural and Construction Aerodynamics (1984).

2. E. Simiu, R. Skanlan, Wind effect on structures (1984).

3. L.E. Sternin, Basics of Gas Dynamics (2016).

4. V.T. Samsonov, Aerodynamics of industrial buildings: aerodynamic calculation during design of ventilation exhausts (2019).

5. T.V. Zolina, P.N.Sadchikov, Vestnik MGSU 9, 15-25 (2016) doi: 10.22227/1997-0935.2016.9.15- 
6. V.N. Gordeev, A.I. Lantukh-Lyashchenko, V.A. Pashinskiy, A.V. Perel'muter, S.F. Puchugin, Loads and Effects on Buildings and Structures (ASV Publ., 2011).

7. Yu.A. Tabunschikov, N.V. Shilkin, AVOK. 8 (2004).

8. S.F. Pichugin, A.V. Makhin ko, Wind Load on Building Structures (Poltava, ASMI Publ., 2005).

9. M. Raffel, Ch.E. Willert, S.T. Wereley, J. Kompenhans, Practical Image Velometry. A Practical Guide (Springer, 2007).

10. V. Akberdina, L. Pushkareva, Key aspects of technological leadership within the context of fourth industrial revolution. 4th International Conference on Social, Business, and Academic Leadership (ICSBAL 2019) (Atlantis Press, 2019)

11. O.O. Egorychev, P.S. Churin, O.I. Poddaeva, Industrial and Civil Engineering 9, 28-30 (2014).

12. O.O. Egorychev, S.V. Guvernyuk, S.A. Isaev, O.I. Poddaeva, N.V. Kornev, A.E. Usachov, Vestnik MGSU 3(1), 185-191 (2011).

13. O.I. Poddaeva, Industrial and Civil Engineering 9, 35-38 (2013).

14. Y.M. Babich, J.-P. Rewinkel, S.V. Filipchyk, Bulletin of Belorussian and Russian University 1(42), 126-135 (2014).

15. S.N. Bulgakov, A.G. Tamrazyan, A.Yu Stepanov, Risk Reduction in Construction during Emergency Situations of Natural and Technogenic Character (Moscow, MAKS Press Publ., 2004).

16. A.I. Gnyrya, S.V. Korobkov, et al., Bulletin of TGASU 4 (2011). 